Commun. Fac. Sci. Univ. Ank. Ser. A1 Math. Stat.

Volume 68, Number 2, Pages 1350-1358(2019)

DOI: $10.31801 /$ cfsuasmas. 529578

ISSN 1303-5991 E-ISSN 2618-6470

http://communications.science.ankara.edu.tr/index.php?series=A1

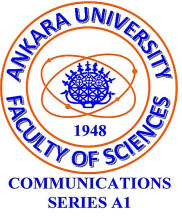

\title{
A NOTE ON DOMINATOR CHROMATIC NUMBER OF LINE GRAPH AND JUMP GRAPH OF SOME GRAPHS
}

\author{
R. KALAIVANI AND D.VIJAYALAKSHMI
}

\begin{abstract}
A dominator coloring is a coloring of the vertices of a graph such that every vertex is either alone in its color class or adjacent to all vertices of at least one other color class. In this paper, we obtain the dominator chromatic number for the Line graph of some graphs, Central graph of Line graph of Star graph and Central graph of Line graph of Double Star graph. And also we obtain the dominator chromatic number for $J\left(S_{n}\right), J\left(C_{n}\right)$ and $J\left(K_{1, n, n, n}\right)$ respectively.
\end{abstract}

\section{Introduction and Preliminaries}

All graphs considered here are finite, undirected and simple graphs. For graph theoretic terminology refer to D. B. West [10]. Let $G$ be a graph, with vertex set $V(G)$ and edge set $E(G)$. A set $D \subseteq V(G)$ is a dominating set if every vertex of $V(G) \backslash D$ has a neighbor in $D$. The domination number $\gamma(G)$ is the minimum cardinality among all the dominating sets of $G$.

A proper coloring of a graph $G$ is a function from the set of vertices of a graph to a set of colors such that any two adjacent vertices have different colors. A subset of vertices colored with the same color is called a color class. The chromatic number is the minimum number of colors needed in a proper coloring of a graph and is denoted by $\chi(G)$.

A dominator coloring of a graph $G$ is a proper coloring of graph such that every vertex of $V$ dominates all vertices of at least one color class (possibly its own class). i.e., it is coloring of the vertices of a graph such that every vertex is either alone in its color class or adjacent to all vertices of at least one other class and this concept was introduced by Ralucca Michelle Gera in 2006 [3] and studied further in [4]

Received by the editors: February 05, 2018; Accepted: June 27, 2018.

2010 Mathematics Subject Classification. 05C15, 05C 75.

Key words and phrases. Coloring, domination, dominator doloring, line graph, jump graph and central graph.

Submitted via International Conference on Current Scenario in Pure and Applied Mathematics [ICCSPAM 2018].

(C)2019 Ankara University Communications Faculty of Sciences University of Ankara-Series A1 Mathematics and Statistics 
and [5] and recently in [2]. The dominator coloring of bipartite graph, central and middle graph of path and cycle graph were also studied in various paper [7, 8,

The central graph 9] $C(G)$ of a graph $G$ is obtained from $G$ by adding a new vertex on each edge of $G$, and then joining each pair of vertices of the original graph which were previously non-adjacent.

The line graph [6] of $G$, denoted by $L(G)$, is a graph whose vertices are the edges of $G$, and if $u, v \in E(G)$ then $u v \in E(L(G))$ if $u$ and $v$ share a vertex in $G$. The Line graph is defined as follows. The Line graph of $G$ denoted by $L(G)$ is the intersection graph of the edges of $G$, representing each edge by the set of its two end vertices. Otherwise $L(G)$ is a graph such that

- Each vertex of $L(G)$ represents an edge of $G$.

- Two vertices of $L(G)$ are adjacent if their corresponding edges share a common end point in $G$.

The jump graph [1] $J(G)$ of a graph $G$ is the graph defined on $E(G)$ and in which two vertices are adjacent if and only if they are not adjacent in $G$. Since both $L(G)$ and $J(G)$ are defined on the edge set of a graph $G$, it follows that isolated vertices of $G$ (if $G$ has ) play no role in line graph and jump graph transformation. We assume that the graph $G$ under consideration is nonempty and has no isolated vertices.

The line graph $L(G)$ of $G$ has the edges of $G$ as its vertices which are adjacent in $L(G)$ if and only if the corresponding edges are adjacent in $G$. We call the complement of line graph $L(G)$ as the jump graph $J(G)$ of $G$, found in [1].

\section{Dominator Chromatic Number of Line Graph of Some Graphs}

Theorem 1. For any n, the dominator chromatic number of Line graph of double star graph is, $\chi_{d}\left(L\left(K_{1, n, n}\right)\right)=n+1$.

Proof. Let $K_{1, n . n}$ be a double star graph. The vertex set of $K_{1, n . n}$ is defined as $V\left(K_{1, n, n}\right)=\{w\} \cup\left\{w_{i}: 1 \leq i \leq n\right\} \cup\left\{u_{i}: 1 \leq i \leq n\right\}$ where $w$ is the root vertex of star graph. Consider $L\left(K_{1, n, n}\right)$, by the definition of line graph, each edge of $K_{1, n, n}$ taken to be as vertex in $L\left(K_{1, n, n}\right)$ namely $\left\{e_{i}: 1 \leq i \leq n\right\} \cup\left\{s_{i}: 1 \leq i \leq n\right\}$. The vertex set of line graph of double star graph is defined as

$$
V\left(L\left(K_{1, n, n}\right)\right)=\left\{e_{i}: 1 \leq i \leq n\right\} \cup\left\{s_{i}: 1 \leq i \leq n\right\} .
$$

Here the vertices $\left\{e_{1}, e_{2}, \ldots, e_{n}\right\}$ induces a clique of order $n$ in $L\left(K_{1, n, n}\right)$.

Now define a coloring $c: V\left(L\left(K_{1, n, n}\right)\right) \rightarrow\{1,2,3, \ldots n+1\}$. The vertex set of $L\left(K_{1, n, n}\right)$ is colored as follows.

$$
c\left(V\left(L\left(K_{1, n, n}\right)\right)\right)=\left\{\begin{array}{l}
i \text { for } e_{i}: 1 \leq i \leq n \\
n+1 \text { for } s_{i}: 1 \leq i \leq n
\end{array}\right.
$$

It is easy to see that above assignment is a dominator coloring with $n+1$ colors. By the definition of dominator coloring the vertex set $s_{i}: 1 \leq i \leq n$ dominates the color class of $e_{i}: 1 \leq i \leq n$ and $e_{i}$ dominates itself. Thus $\chi_{d}\left(L\left(K_{1, n, n}\right)\right) \leq$ 
$n+1$. Let us assume that $\chi_{d}\left(L\left(K_{1, n, n}\right)\right)$ is lesser than $\mathrm{n}+1$, i.e., $\chi_{d}\left(L\left(K_{1, n, n}\right)=n\right.$. Since $e_{i}: 1 \leq i \leq n$ forms a clique of order $n$ so we have to assign $n$ colors to $e_{i}$. To obtain minimum number of coloring, if we assign any preused colors from $e_{i}: 1 \leq i \leq n$ to the vertices of $s_{i}: 1 \leq i \leq n$ then it contradicts the definition of dominator coloring. Therefore dominator coloring with $n$ colors is not possible. Hence $\chi_{d}\left(L\left(K_{1, n, n}\right)\right)=n+1$.

Example 2. The Dominator coloring of $L\left(K_{1,5,5}\right)$ is shown in the following Figure 1

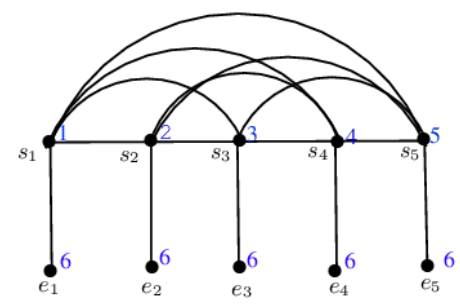

Figure 1. $\chi_{d}\left(L\left(K_{1,5,5}\right)\right)$

Theorem 3. For any n, the dominator chromatic number of Line graph of Bi-star graph is

$$
\chi_{d}\left(L\left(B_{n, n}\right)\right)=n+1 .
$$

Proof. Consider the Bistar $B_{n, n}$. By definition of Bistar, let $\left\{u_{i}: 1 \leq i \leq n\right\}$ be the $n$ pendant edges attached to the vertex $u$ and $\left\{v_{i}: 1 \leq i \leq n\right\}$ be the another $n$ pendant edges attached to the vertex $v$. Consider the Line graph of $B_{n, n}$. By the definition of Line graph, the edge set of Bi-star corresponds to the vertex set of $L\left(B_{n, n}\right)$. The vertex set is defined as

$$
V\left(L\left(B_{n, n}\right)\right)=\{w\} \cup\left\{s_{i}: 1 \leq i \leq n\right\} \cup\left\{e_{i}: 1 \leq i \leq n\right\} .
$$

In $L\left(B_{n, n}\right)$ the vertices $s_{i}: 1 \leq i \leq n$ along with $w$ forms a complete graph of order $n+1$. Also we see that the vertices $e_{i}: 1 \leq i \leq n$ together with $w$ forms another complete graph of order $n+1$.

Now consider the color class $C=\left\{c_{1}, c_{2}, c_{3}, \ldots, c_{n}\right\}$. For $1 \leq i \leq n$, assign $c_{i}$ colors to $e_{i}$ and $s_{i}$. Thus to make the coloring as dominator chromatic one, we should assign only the same set of colours to $e_{i}: 1 \leq i \leq n$ which we already assigned for $s_{i}: 1 \leq i \leq n$. Since $e_{i}$ and $s_{i}$ forms a complete graph along with $w$ so we have to assign $c_{n+1}$ colors to $w$. Now all the vertices $s_{i}, e_{i}: 1 \leq i \leq n$ and $w$ realizes its own colour, which produces a dominator coloring. Thus by the dominator coloring procedure the above said coloring is dominator chromatic. Hence $\chi_{d}\left(L\left(B_{n, n}\right)\right)=n+1$. 
Theorem 4. Let $m, n \geq 5$. The dominator chromatic number of the Line graph of Bipartite graph is,

$$
\chi_{d}\left(L\left(K_{m, n}\right)\right)=\left\{\begin{array}{l}
m+n-1 \text { if } m<n \\
2 m \text { if } m=n \\
m+n-1 \text { if } m>n .
\end{array}\right.
$$

Proof. Let $K_{m, n}$ be the Complete Bi-partite graph with bipartition $(X, Y)$ where $X=\left\{v_{i}: 1 \leq i \leq n\right\}$ and $Y=\left\{u_{i}: 1 \leq i \leq n\right\}$. Consider the Line graph of $K_{m, n}$. Let $v_{i j}$ be the edge between the vertex $v_{i}$ and $u_{j}$ for $i=1,2,3, \ldots, m, j=1,2,3, \ldots, n$ i.e. $v_{i} u_{j}=v_{i j}: 1 \leq i \leq m, 1 \leq j \leq n$. By the definition of the Line graph, edges in $K_{m, n}$ corresponds to the vertices in $L\left(K_{m, n}\right)$ i.e.

$$
V\left[L\left(K_{m, n}\right)\right]=\left\{v_{i j}: 1 \leq i \leq m, 1 \leq j \leq n\right\} .
$$

Note that for each $i$, we say that $\left\langle v_{i j}: 1 \leq j \leq n>\right.$ is a complete graph of order $n$. Also we say for each $j,\left\langle v_{i j}: 1 \leq i \leq m>\right.$ forms a complete graph of order $m$. Clearly the number of cliques in $L(K m, n)$ is $m+n$.

Case 1. when $m<n$

Now consider the color class $C=\left\{c_{1}, c_{2}, c_{3}, \ldots, c_{m+n}, c_{m+n-1}\right\}$. Assign the color $c_{i}$ to $v_{i 1}$ for $1 \leq i \leq m$. Next assign color $c_{m+n-j+1}$ to the vertices in the first row of $v_{1 j}$, for $2 \leq j \leq n$ and assign this same colors to each row of $v_{i j}$, for $2 \leq i \leq m, 2 \leq j \leq n$ in anti clockwise manner. The vertices $v_{i 1}$ dominates any one color class of $v_{i 1}$ and the remaining vertices $v_{i j}: 2 \leq i \leq m, 1 \leq j \leq n$ dominates the color class $v_{i 1}: 1 \leq i \leq m$. By the observation an easy check shows that $\chi_{d}\left(B_{m, n}\right)=m+n-1$.

Case 2. when $m=n$

Assign the color $c_{i}$ to $v_{1 j}, 1 \leq j \leq n$. Next assign the color $c_{2 m-j+1}$ to $v_{2 j}$ for $1 \leq j \leq n$ and assign this same colors to each row $v_{i j}$ for $3 \leq i \leq m, 1 \leq j \leq n$ in anti clockwise rotation. By the definition of dominator coloring, here the vertices $v_{1 j}$ dominates at least any one of the color class $c_{i}$ and the remaining vertices $v_{i j}: 2 \leq i \leq m, 1 \leq j \leq n$ dominates the color class of $v_{1 j}$ for $2 \leq j \leq n$. Hence an easy observation shows that $\chi_{d}\left(B_{m, n}\right)=2 m$.

Case 3. when $m>n$

Assign the color $c_{i}$ to $v_{1 j}$ for $1 \leq j \leq n$. Next assign color $c_{m+n-i+1}$ to $v_{i 1}$ for $2 \leq i \leq m$ and assign this same colors to each column in anti clockwise manner $v_{i j}$ for $3 \leq i \leq m, 1 \leq j \leq n$ in anti clockwise rotation. By the definition of dominator coloring, the vertices $v_{1 j}: 1 \leq j \leq n$ dominate any one color class of $v_{1 j}$ and the remaining vertices $v_{i j}: 2 \leq 1 \leq m, 1 \leq j \leq n$ dominate the color class vi1 $: 1 \leq i \leq m$. By the observation an easy check shows that $\chi_{d}\left(B_{m, n}\right)=m+n-1$. This completes the proof of the theorem.

Theorem 5. For the Line graph of $K_{1, n}, \chi_{d}\left(C\left(L\left(K_{1, n}\right)\right)\right)=n+1$.

Proof. Consider the star graph of $K_{1, n}$. Let $V\left(K_{1, n}\right)=\{v\} \cup\left\{v_{i}: 1 \leq i \leq n\right\}$. By the definition of the Line graph, edge set in $K_{1, n}$ corresponds to the vertex 
set of $K_{1, n}$ i.e. $V\left(L\left(K_{1, n}\right)\right)=\left\{e_{i}: 1 \leq i \leq n\right\}$. Here in $L\left(K_{1, n}\right)$, the vertices $\left\{e_{i}: 1 \leq i \leq n\right\}$ forms a clique of order $n$.

Next consider the Central graph of Line graph of the star graph . By the definition of central graph, $C\left(L\left(K_{1, n}\right)\right)$ is obtained by subdividing each edge $e_{i}$ exactly only once and joining all the non-adjacent vertices of $L\left(K_{1, n}\right)$ in $C\left(L\left(K_{1, n}\right)\right)$. The vertex set is defined as,

$$
V\left(C\left(L\left(K_{1, n}\right)\right)=\left\{e_{i}: 1 \leq i \leq n\right\} \cup\left\{e_{i}^{\prime}: 1 \leq i \leq n(n-1) / 2\right\}\right.
$$

Now consider the color class $C=\left\{c_{1}, c_{2}, c_{3}, \ldots, c_{n+1}\right\}$. For $1 \leq i \leq n$, assign the color $c_{i}$ to $e_{i}$ and assign the color $c_{n+1}$ to $e_{i}^{\prime}: 1 \leq i \leq n(n-1) / 2$. Here every vertex in $e_{i}^{\prime}: 1 \leq i \leq n(n-1) / 2$ dominates atleast any one of the color class of $e_{i}: 1 \leq i \leq n$ and all the vertices of $e_{i}$ dominates itself. Hence an easy check shows that $\chi_{d}\left(C\left(L\left(K_{1, n}\right)\right)\right)=n+1$.

Theorem 6. Let $n \geq 2$. The dominator chromatic number of Central graph of Line graph of $K_{1, n, n}$ is,

$$
\chi_{d}\left(C\left(L\left(K_{1, n, n}\right)\right)\right)=\left\{\begin{array}{l}
3\lfloor n / 2\rfloor+1 \text { if } n \text { is even } \\
3\lfloor n / 2\rfloor+2 \text { if } n \text { is odd }
\end{array}\right.
$$

Proof. Consider the star graph of $K_{1, n, n}$. Let

$$
V\left(K_{1, n, n}\right)=\{w\} \cup\left\{w_{i}: 1 \leq i \leq n\right\} \cup\left\{w_{i}^{\prime}: 1 \leq i \leq n\right\} .
$$

By the definition of the Line graph, edge set in $K_{1, n, n}$ corresponds to the vertex set of $K_{1, n, n}$ i.e. $V\left(L\left(K_{1, n, n}\right)\right)=\left\{v_{i}: 1 \leq i \leq n\right\} \cup\left\{u_{i}: 1 \leq i \leq n\right\}$. Now consider the Central graph of Line graph of the star graph. By the definition of central graph $C\left(L\left(K_{1, n, n}\right)\right)$ is obtained by subdividing each edge $v_{i}, u_{i}: 1 \leq i \leq n$ exactly only once and joining all the non-adjacent vertices of $L\left(K_{1, n, n}\right)$ in $C\left(L\left(K_{1, n, n}\right)\right)$. The vertex set is defined as

$$
\begin{aligned}
V\left(C\left(L\left(K_{1, n, n}\right)\right)\right)= & \left\{v_{i}: 1 \leq i \leq n\right\} \cup\left\{u_{i}: 1 \leq i \leq n\right\} \cup\left\{e_{i}: 1 \leq i \leq n(n-1) / 2\right\} \\
& \cup\left\{e_{i}^{\prime}: 1 \leq i \leq n\right\} .
\end{aligned}
$$




\section{Case 1. when $n$ is even.}

Now we define coloring $c: V\left[C\left(L\left(K_{1, n, n}\right)\right)\right] \rightarrow\{1,2,3, \ldots, 3\lfloor n / 2\rfloor+1\}$. The vertex set of $C\left(L\left(K_{1, n, n}\right)\right)$ is colored as follows.

$$
c\left[V\left(C\left(L\left(K_{1, n, n}\right)\right)\right)\right]=\left\{\begin{array}{l}
i \text { for } u_{i}: 1 \leq i \leq n \\
n+i / 2 \text { for } v_{i} \text { where } i=2,4,6, \ldots, n \\
2 \text { for } e_{i}: 1 \leq i \leq n(n-1) / 2, i \neq 2, \\
3 \text { for } e_{2} \\
2 \text { for } e_{i}^{\prime}: 1 \leq i \leq n \\
3\lfloor n / 2\rfloor+1 \text { for } v_{i} \text { where } i=1,3,5, \ldots, n-1
\end{array}\right.
$$

Thus the above coloring assignment is a dominator coloring with $3\lfloor n / 2\rfloor+1$ colors. By the definition of dominator coloring, every vertex in $u_{i}: 1 \leq i \leq n$ dominates atleast any one of the color class of $u_{i}: 1 \leq i \leq n$ and all the vertices of $v_{i}, e_{i}$ and $e_{i}^{\prime}$ are dominated by the atleast anyone of the color class of $u_{i}: 1 \leq i \leq n$. or $e_{i}: 1 \leq$ $i \leq n(n-1) / 2$. Hence an easy check shows that $\chi_{d}\left(C\left(L\left(K_{1, n, n}\right)\right)\right)=3\lfloor n / 2\rfloor+1$.

Case 2. when $n$ is odd

Now we define coloring

$$
c: V\left[C\left(L\left(K_{1, n, n}\right)\right)\right] \rightarrow\{1,2,3, \ldots, 3\lfloor n / 2\rfloor+2\} .
$$

The vertex set of $C\left(L\left(K_{1, n, n}\right)\right)$ is colored as follows.

$$
c\left[V\left(C\left(L\left(K_{1, n, n}\right)\right)\right)\right]=\left\{\begin{array}{l}
i \text { for } u_{i}: 1 \leq i \leq n \\
n+i / 2 \text { for } v_{i} \text { where } i=2,4,6, \ldots, n-1 \\
2 \text { for } e_{i}: 1 \leq i \leq n(n-1) / 2, i \neq 2, \\
3 \text { for } e_{2} \\
2 \text { for } e_{i}^{\prime}: 1 \leq i \leq n \\
3\lfloor n / 2\rfloor+2 \text { for } v_{i} \text { where } i=1,3,5, \ldots, n
\end{array}\right.
$$

It is easy to see that above coloring assignment is a dominator coloring with $3\lfloor n / 2\rfloor+2$ colors. By the definition of dominator coloring here every vertex in $u_{i}$ : $1 \leq i \leq n$ dominates the any one color class of $u_{i}: 1 \leq i \leq n$ and all the vertices of $v_{i}, e_{i}$ and $e_{i}^{\prime}$ dominates atleast any one the color class of $u_{i}$ or $v_{i}: 1 \leq i \leq n(n-1) / 2$. Hence an easy observation shows that $\chi_{d}\left(C\left(L\left(K_{1, n, n}\right)\right)\right)=3\lfloor n / 2\rfloor+2$.

\section{Dominator Chromatic Number of Jump Graph of Some Graphs}

Theorem 7. Let $n \geq 4$, the dominator chromatic number of Jump graph of Sunlet graph is, $\chi_{d}\left(J\left(S_{n}\right)\right)=n$.

Proof. Let us define the vertex set $V$ of $S_{n}$ as $V\left(S_{n}\right)=\left\{v_{i}: \leq i \leq n\right\} \cup\left\{u_{i}: 1 \leq i \leq n\right\}$ where $v_{i}$ are the vertices of cycles taken in cyclic order and $u_{i}$ are the pendent vertices. By the definition of jump graph, the vertex set are defined as $V\left(J\left(S_{n}\right)\right)=$ 
$\left\{e_{i}: 1 \leq i \leq n\right\} \cup\left\{e_{i}^{\prime}: 1 \leq i \leq n\right\}$. Now we define coloring

$$
c: V\left[J\left(S_{n}\right)\right] \rightarrow\{1,2,3, \ldots, n .\} .
$$

The vertex set of $J\left(S_{n}\right)$ is colored as follows.

$$
c\left[V\left(J\left(S_{n}\right)\right)\right]=\left\{\begin{array}{l}
i \text { for } e_{i}^{\prime}: 1 \leq i \leq n \\
i+1 \text { for } e_{i}: 1 \leq i \leq n-1 \\
1 \text { for } e_{n} .
\end{array}\right.
$$

It is not hard to see that above assignment is dominator coloring with $n$ colors. Here every vertex in $e_{i}, e_{i}^{\prime}$ dominates atleast any of the color class of $e_{i}: 1 \leq i \leq n$ and $e_{i}^{\prime}: 1 \leq i \leq n$. On the other hand we can not assign $n-1$ colors to $e_{i}^{\prime}$ because $e_{i}^{\prime}: 1 \leq i \leq n$ forms a clique of order $n$. Hence $\chi_{d}\left(J\left(S_{n}\right)\right)=n$.

Theorem 8. Let $n \geq 3$, the dominator chromatic number of Jump graph of Triple star graph is, $\chi_{d}\left(J\left(K_{1, n, n, n}\right)\right)=n+1$.

Proof. Let us define the vertex set $V$ of $K_{1, n, n, n}$ as

$$
\begin{aligned}
V\left(K_{1, n, n, n}\right)= & \{w\} \cup\left\{w_{i}: 1 \leq i \leq n\right\} \cup\left\{w_{i}^{\prime}: 1 \leq i \leq n\right\} \\
& \cup\left\{w_{i}^{\prime \prime}:: 1 \leq i \leq n\right\} .
\end{aligned}
$$

By the definition of jump graph, the vertex set of $J\left(K_{1, n, n, n}\right)$ is defined as

$$
V\left[J\left(K_{1, n, n, n}\right)\right]=\left\{v_{i}: 1 \leq i \leq n\right\} \cup\left\{u_{i}: 1 \leq i \leq n\right\} \cup\left\{e_{i}: 1 \leq i \leq n\right\} .
$$

Now we define coloring $c: V\left(J\left(K_{1, n, n, n}\right)\right) \rightarrow\{1,2,3, \ldots, n, n+1\}$.

The vertex set of $V\left(J\left(K_{1, n, n, n}\right)\right)$ is colored as follows.

$$
c\left[V\left(J\left(K_{1, n, n, n}\right)\right]=\left\{\begin{array}{l}
i \text { for } e_{i}, u_{i}: 1 \leq i \leq n \\
n+1 \text { for } v_{i}: 1 \leq i \leq n
\end{array}\right.\right.
$$

The above assignment gives lead to be a dominator coloring with $n+1$ colors. By the definition of dominator coloring, every vertex in $v_{i}: 1 \leq i \leq n$ dominates atleast any one of the color classes of $e_{i}, u_{i}: 1 \leq i \leq n$. On the other hand, if we assign $n$ colors to $u_{i}, v_{i}, e_{i}$ then the sub graph of atleast any two vertices receives the same colors. Therefore an easy check shows that dominator coloring with $n$ color is not possible. Hence $\chi_{d}\left(J\left(K_{1, n, n, n}\right)\right)=n+1$.

Theorem 9. Let $n \geq 3$, the dominator chromatic number of Jump graph of cycle graph is

$$
\chi_{d}\left(J\left(C_{n}\right)\right)=\lceil n / 2\rceil .
$$


Proof. Let $V\left(C_{n}\right)=\left\{v_{i}: 1 \leq i \leq n\right\}$. By the definition, of jump graph the vertex set is defined as $V\left(J\left(\left(C_{n}\right)\right)=\left\{u_{i}: 1 \leq i \leq n\right\}\right.$ Now we define coloring $c$ : $V\left[J\left(C_{n}\right)\right] \rightarrow\{1,2,3, \ldots,\lceil n / 2\rceil\}$. The vertex set of $V\left(J\left(C_{n}\right)\right)$ is colored as follows.

$$
c\left[V\left(J\left(C_{n}\right)\right)\right]=\left\{\begin{array}{l}
\lceil i / 2\rceil \text { for } u_{i} \text { when } i=1,3,5, \ldots, 2 k-1,1 \leq k \leq\lceil n / 2\rceil \\
i / 2 \text { for } u_{i} \text { when } i=2,4,6, \ldots, 2 k, 1 \leq k \leq\lceil n / 2\rceil
\end{array}\right.
$$

Thus the above coloring assignment leads to be a dominator coloring with $n$ colors. By the definition of dominator coloring every vertex in $u_{i}: 1 \leq i \leq n$ dominates at least any one color class of $u_{i}$ Thus $\chi_{d}\left(J\left(C_{n}\right)\right) \leq\lceil n / 2\rceil$.

To prove $\chi_{d}\left(J\left(C_{n}\right)\right) \geq\lceil n / 2\rceil$. Let us assume that $\chi_{d}\left(J\left(C_{n}\right)\right)$ is lesser than $\lceil n / 2\rceil$ If we assign less than $\lceil n / 2\rceil$ colors to $u_{i}$ then a subgraph of atleast any two of the vertices receives same color and it contradicts the definition of dominator coloring - Hence an easy observation shows that dominator coloring with less than $\lceil n / 2\rceil$ color is not possible. Hence $\chi_{d}\left(J\left(C_{n}\right)\right)=\lceil n / 2\rceil$.

Conclusion 10. In this paper, we obtained the dominator chromatic number for the Central graph of Line graph of Star graph and Central graph of Line graph of Double Star graph, also the dominator chromatic number for Jump graph of some graphs such as $J\left(S_{n}\right), J\left(C_{n}\right)$ and $J\left(K_{1, n, n, n}\right)$ Graphs are obtained. This paper can further be extended by identifying graph families of graphs for which these chromatic numbers are equal to other kinds of chromatic numbers.

\section{REFERENCES}

[1] Chartrand, G., Hevia, H.E., Jarrett, B. and Schultz, M., Subgraph distances in graphs defined by edge transfers, Discrete Math, 170 (1997), 63-79.

[2] Chellali, M. and Maffray, F., Dominator Colorings in Some Classes of Graphs, Graphs Combin, 28 (2012), 97-107.

[3] Gera, R., Horton, S. and Rasmussen, C., Dominator Colorings and Safe Clique Partitions, Congressus Numerantium, (2006), 19-32.

[4] Gera, R., On The Dominator Colorings in Bipartite Graphs in: Proceedings of the 4th International Conference on Information Technology: New Generations (2007), 947-952.

[5] Gera, R.M, On Dominator Coloring in Graphs, Graph Theory Notes N.Y. LII, (2007), 947 -952 .

[6] Haynes, T.W., Hedetniemi, S.T. and Slater, P.J, Fundamentals of Domination in Graphs, Marcel Dekker., New york, 1998.

[7] Kavitha, K. and David, N.G., Dominator Coloring of Central Graphs, International Journal of Computer Applications, 51(12), (2012), 11-14.

[8] Kavitha, K. and David, N. G., Dominator Coloring on Star and Double Star Graph Families, International Journal of Computer Applications, 48(3), (2012), 22-25.

[9] Vijayalakshmi, D., Study on $b$-Chromatic Colouring of Graphs,Ph.D Thesis, Bharathiar University, Coimbatore, India, (2012).

[10] West, D. B., Introduction to Graph Theorey, 2nd ed., Prentice Hall, USA, 2001. 
Current address: R. Kalaivani: Department of Mathematics, Kongunadu Arts and Science College, Coimbatore - 641 029, Tamil Nadu, India

E-mail address: kalaivanirm@yahoo.com

ORCID Address: http://orcid.org/0000-0002-6751-8985

Current address: D.Vijayalakshmi: Department of Mathematics, Kongunadu Arts and Science College, Coimbatore - 641 029, Tamil Nadu, India

E-mail address: vijikasc@gmail.com

ORCID Address: http://orcid.org/0000-0002-8925-1134 